\title{
Muscimol Inactivation of the Dorsal Hippocampus Impairs Contextual Retrieval of Fear Memory
}

\author{
William Holt ${ }^{1}$ and Stephen Maren ${ }^{1,2}$ \\ ${ }^{1}$ Department of Psychology and 2Neuroscience Program, University of Michigan, Ann Arbor, Michigan 48109-1109
}

Some models of hippocampal function have suggested a role of the hippocampus in contextual memory retrieval. We have examined this hypothesis by assessing the impact of reversible inactivation of the dorsal hippocampus $(\mathrm{DH})$ on the contextspecific expression of latent inhibition, a decrement in conditional responding produced by preexposure to a to-beconditional stimulus. In Experiment 1, rats received tone preexposure either in the context that would later be used for extinction testing (context A) or in a different context (context C); a third group of rats did not receive tone preexposure. All rats then received fear conditioning, which consisted of tonefootshock pairings, in a third distinct context (context B). The following day conditional fear to the tone was assessed in one of the preexposure contexts (context $A$ ) by measuring freezing during a tone extinction test. Rats preexposed and tested in the same context exhibited less freezing to the tone than either rats preexposed and tested in different contexts or nonpreexposed rats. These results indicate that the expression of latent inhibition is context specific. In Experiment 2, DH inactivation eliminated the context-specific expression of latent inhibition. Compared with saline-infused rats, rats infused with muscimol into the $\mathrm{DH}$ exhibited low levels of tone freezing independent of whether they had received tone preexposure in the test context or in a different context. Experiment 3 revealed normal contextual discrimination in rats after $\mathrm{DH}$ inactivation. These results suggest the $\mathrm{DH}$ is required for contextual memory retrieval in a latent inhibition paradigm.

Key words: Pavlovian fear conditioning; latent inhibition; auditory conditioning; hippocampus; context; muscimol; freezing; rats
Recently, there has been increasing interest in the role of the hippocampal formation in the neural substrates of contextual learning. Pavlovian fear conditioning has proved to be a profitable behavioral model for studying the role of the hippocampus in contextual learning (Maren et al., 1998; Holland and Bouton, 1999). In Pavlovian fear conditioning, a neutral conditional stimulus (CS), such as a tone, is paired with an aversive unconditional stimulus (US), such as a footshock. After a few pairings, the CS comes to elicit a number of conditional fear responses including potentiated acoustic startle, increased blood pressure, and freezing. To date, core efforts in this paradigm have been directed at examining the role of the hippocampal system in mediating contextual fear conditioning (e.g., Kim and Fanselow, 1992; Phillips and LeDoux, 1992; Anagnostaras et al., 1999). In addition to its role in contextual fear conditioning, the hippocampus has been implicated in contextual memory retrieval (e.g., Hirsh, 1974, 1980), the process by which performance of learned behavior is facilitated to the degree that the context of performance resembles the context of learning (Tulving and Thomson, 1973). In Pavlovian paradigms, contextual retrieval is especially important when a CS acquires two or more conflicting meanings, which is the case when rats experience both conditioning (CS-US) and extinction (CS-“no US”) (e.g., Bouton, 1994).

In studies examining the contribution of the hippocampus to

\footnotetext{
Received June 7, 1999; revised July 23, 1999; accepted July 28, 1999.

This research was supported by National Institute of Mental Health Grants MH57865 and MH57360 to S.M. W.H. was supported by a National Science Foundation graduate student fellowship. We would like to thank Rishi Gupta for technical assistance.

Correspondence should be addressed to Dr. Stephen Maren, Department of Psychology, University of Michigan, 525 East University Avenue, Ann Arbor, MI 48109-1109.

Copyright (C) 1999 Society for Neuroscience $\quad 0270-6474 / 99 / 199054-09 \$ 05.00 / 0$
}

contextual retrieval, permanent pretraining lesions have been used (Good and Honey, 1991; Honey and Good, 1993). Although these studies suggest that hippocampal lesions impair contextual memory retrieval, pretraining lesions necessarily confound the hippocampal role in retrieval processes with its possible role in encoding processes. To overcome these problems, we have used a reversible hippocampal lesion technique together with a Pavlovian paradigm that isolates contextual retrieval. We targeted contextual retrieval in latent inhibition, which is a decrement in conditional responding produced by CS preexposure (Lubow, 1973). Latent inhibition is context specific. Conditional responding is diminished when both training and testing occur in the context of CS preexposure but not when the conditioning context is different from the preexposure context (e.g., Hall and Minor, 1984). Importantly, however, latent inhibition can be "renewed" when retrieval testing occurs in the preexposure context (Dexter and Merrill, 1969; Wright et al., 1986; Bouton and Swartzentruber, 1989). On the basis of these results, Bouton (1993) has suggested that a contextual retrieval process determines whether the CS-US memory acquired during training or the CS-"no event" memory acquired during preexposure is expressed during testing.

In the present experiments, we have used this latent inhibition paradigm along with reversible inactivation of the dorsal hippocampus $(\mathrm{DH})$ to examine the role of the $\mathrm{DH}$ in contextual memory retrieval. We first established that the expression of latent inhibition is context specific in our conditional-freezing paradigm. We then examined the impact of reversible inactivation of the $\mathrm{DH}$ on the context-specific expression of latent inhibition. Finally, we examined the effect of DH inactivation on a contextual discrimination. Our results reveal an important role of the $\mathrm{DH}$ in the contextual retrieval of fear memories. 


\section{EXPERIMENT 1: IS THE EXPRESSION OF LATENT INHIBITION CONTEXT SPECIFIC?}

Bouton and Swartzentruber (1989) reported previously that the expression of latent inhibition is context specific in a fearconditioning paradigm. They used bar-press suppression as an index of conditional fear. In the present experiment, we sought to determine whether CS preexposure would also produce contextspecific expression of latent inhibition assessed by another fear response, conditional freezing [somatomotor immobility (Fanselow, 1980)]. Rats were preexposed to tones in one of two contexts, trained with tone-footshock pairings in a third context, and then returned to one of the preexposure contexts for testing with nonreinforced tone presentation. For comparison, a control group spent equal time in the preexposure contexts but did not receive $\mathrm{CS}$ preexposure. Because the expression of latent inhibition is context specific, we expected that latent inhibition would only be manifest in rats that were preexposed to the tone in the testing context.

\section{MATERIALS AND METHODS}

Subjects. The subjects were 48 adult male Long-Evans rats (200-224 gm) obtained from a commercial supplier (Harlan Sprague Dawley, Indianapolis, IN). After arrival, the rats were housed individually in stainless steel hanging cages on a 14:10 hr light/dark cycle (lights on at 7:00 A.M.) and were fed food and water ad libitum. After being housed, rats were handled (10-20 sec per rat per day) for $5 \mathrm{~d}$ to acclimate them to the experimenter.

Behavioral apparatus. Eight identical observation chambers $(30 \times 24 \times$ $21 \mathrm{~cm}$; MED-Associates, Burlington, VT) were used in the preexposure phase as context A. The chambers were constructed from aluminum (side walls) and Plexiglas (rear wall, ceiling, and hinged front door) and were situated in sound-attenuating cabinets located in a brightly lit and isolated room. The floor of each chamber consisted of 19 stainless steel rods (4 mm in diameter) spaced $1.5 \mathrm{~cm}$ apart (center to center). Rods were wired to a shock source and solid-state grid scrambler (MED-Associates) for the delivery of footshock USs. A speaker mounted outside a grating in one wall of the chamber was used for the delivery of acoustic CSs. A $15 \mathrm{~W}$ house light was mounted on the opposite wall. The chambers were cleaned with a $5 \%$ ammonium hydroxide solution, and stainless steel pans containing a thin film of the same solution were placed underneath the grid floors before the rats were placed inside. Ventilation fans in each chest supplied background noise ( $65 \mathrm{~dB}$, A scale).

The second preexposure context, context $\mathrm{C}$, was comprised of eight identical paint buckets ( 5 gallons; $36 \mathrm{~cm}$ tall $\times 30 \mathrm{~cm}$ in diameter). The bucket bottoms were lined with pine-shaving bedding. The buckets were placed on the floor in the same room that housed the chambers described above. When the room was used as context $\mathrm{C}$, the chamber fans and house lights were turned off, the chambers were not scented with ammonium hydroxide, and the room lighting was dimmed by turning off half of the ceiling lights.

Procedure. Rats were randomly assigned to groups ( $n=16$ per group) that were preexposed to the tone $(10 \mathrm{sec} ; 80 \mathrm{~dB} ; 5 \mathrm{kHz})$ in either context A (PRE-SAME) or context C (PRE-DIFF); a third group of rats did not receive tone preexposure (NO PRE). The preexposure phase lasted $5 \mathrm{~d}$. On each day, each rat spent 38 min in both context $A$ and context $C$; the order of the context exposure was counterbalanced. In the tone preexposure context, rats received 30 tone presentations (60 sec interstimulus interval) $3 \mathrm{~min}$ after placement in the context. Approximately $4 \mathrm{hr}$ lapsed between placement in the two contexts each day. Speakers from the eight conditioning chambers generated tones for both tone-preexposed groups. Tone intensity was adjusted to $90 \mathrm{~dB}$ during context $\mathrm{C}$ sessions (this yielded an $80 \mathrm{~dB}$ tone in the buckets) and $80 \mathrm{~dB}$ during the context $\mathrm{A}$ sessions.

On the sixth day, rats were conditioned in a novel context, context B, with tone-footshock pairings. Context $\mathrm{B}$ consisted of the same chambers used for context A; however all of the ceiling lights and chamber house lights were turned off (illumination in the room was provided by a $40 \mathrm{~W}$ red light). Additionally, the doors on the sound-attenuating cabinets were closed, the ventilation fans were turned off, and the chambers were cleaned with a $1 \%$ acetic acid solution. Rats were transported in squads of eight and placed in the conditioning chambers; chamber position was counterbalanced for each squad and group. The rats received five tone $(10 \mathrm{sec} ; 80 \mathrm{~dB} ; 5 \mathrm{kHz})$-footshock $(1 \mathrm{sec} ; 0.5 \mathrm{~mA})$ trials $(69 \mathrm{sec}$ intertrial interval) 3 min after being placed in the chambers. Sixty seconds after the final shock, the rats were returned to their home cages. Twenty-four hours after the conditioning session, all rats were returned to context $\mathrm{A}$ for an 8 min tone extinction test. For this test, the tone that was paired with shock during conditioning was presented continuously for $8 \mathrm{~min}$; tone onset occurred 2 min after the rats were placed in the chambers. Note that the test context (context A) was the same as the context of CS preexposure for one group of rats (PRE-SAME) but different from the context of CS preexposure for another group of rats (PRE-DIFF).

Fear to the tone CS was assessed by measuring freezing behavior (see Maren, 1998). Each conditioning chamber rested on a load-cell platform that was used to record chamber displacement in response to each rat's motor activity. To insure interchamber reliability, we calibrated each load-cell amplifier to a fixed chamber displacement. The output of each chamber's load cell was set to a gain (vernier dial, 8) that was optimized for detecting freezing behavior. Load-cell amplifier output $(-10$ to +10 V) from each chamber was digitized and acquired on-line using Threshold Activity software (MED-Associates). Absolute values of the load-cell voltages were computed. These values were multiplied by 10 to yield a load-cell activity scale that ranged from 0 to 100 .

For each chamber, load-cell activity was digitized at $5 \mathrm{~Hz}$, yielding one observation per rat every $200 \mathrm{msec}$ ( 300 observations per rat per minute). In all experiments, freezing was quantified by computing the number of observations for each rat that had a value less than the freezing threshold (load-cell activity $=5$; animals exhibit freezing when load-cell activity is at or below this value). To avoid counting momentary inactivity as freezing, we scored an observation as freezing only if it fell within a contiguous group of at least five observations that were all less than the freezing threshold. Thus, freezing was only scored if the rat was immobile for at least $1 \mathrm{sec}$. For each session, the freezing observations were transformed to a percentage of total observations.

Data analysis. For each session, the freezing data were transformed to a percentage of the total observations, a probability estimate that is amenable to analysis with parametric statistics. These probability estimates of freezing were analyzed using ANOVA. Post hoc comparisons in the form of Fisher's PLSD tests were performed after a significant omnibus $F$-ratio. All data are represented as means \pm SEMs. Technical problems during the preexposure phase forced the exclusion of seven rats, which yielded the following groups: NO PRE $(n=13)$, PRE-SAME $(n=14)$, and PRE-DIFF $(n=14)$.

\section{RESULTS}

Freezing during each minute of the tone extinction test in context A is shown in Figure $1 A$. Figure $1 B$ shows the tone-freezing data collapsed across the 8 min tone. To summarize the groups again, PRE-SAME rats were preexposed to the tone in the test context (context A), PRE-DIFF rats were preexposed to the tone in a different context (context C), and NO PRE rats were not preexposed to the tone. As shown, rats in the PRE-SAME condition displayed less conditional freezing to the tone than that exhibited by rats in either the NO PRE or PRE-DIFF conditions. This observation was confirmed by a significant main effect of group $\left[F_{(2,38)}=3.3 ; p<0.05\right]$ in the ANOVA performed on the mean tone-freezing data displayed in Figure $1 B$. Post hoc comparisons $(p<0.05)$ indicated that rats in the PRE-SAME condition froze significantly less than did rats in either the NO PRE or PREDIFF groups, which did not differ from each other. There were no group differences in freezing behavior before tone onset $\left[F_{(2,38)}=0.23 ; p>0.75\right]$.

These results reveal that the expression of latent inhibition is context specific and that latent inhibition is renewed when rats that have been trained in a different context are tested in the context of CS preexposure. Our results also reveal that latent inhibition is not merely a consequence of CS preexposure (see PRE-DIFF group); the expression of latent inhibition required that both CS preexposure and testing occur in the same context, as in the PRE-SAME group. These results suggest that latent 


\section{(A) Tone extinction test}

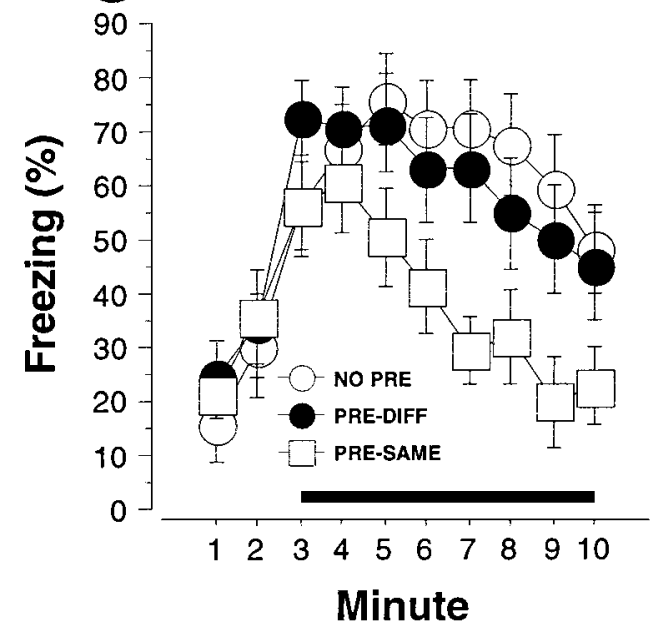

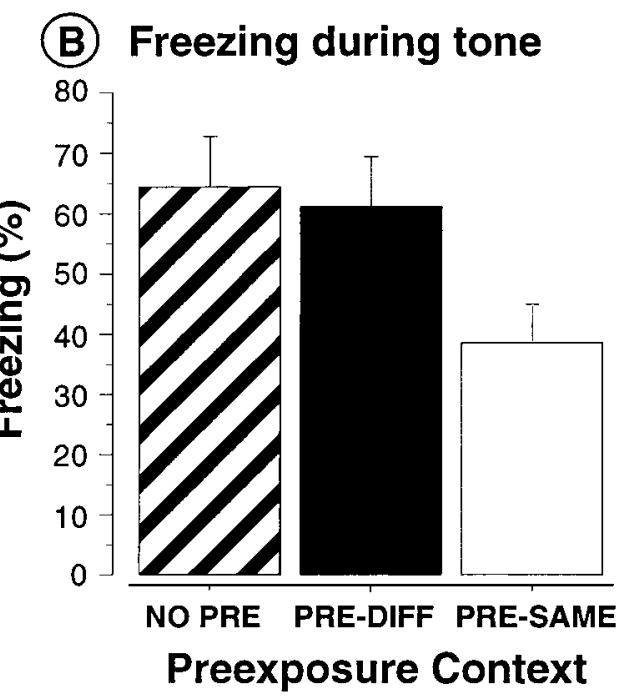

Figure 1. Context-specific expression of latent inhibition (Experiment 1). A, Mean ( \pm SEM) percentage of freezing during the tone extinction test (in context A) conducted $1 \mathrm{~d}$ after conditioning in rats that received CS preexposure in the context that is the same as (PRE-SAME, open squares) the context of later testing or different from (PRE-DIFF, filled squares) the testing context or that received no CS preexposure (NO PRE, open circles). Tone onset occurred at the start of the third minute of the test. $B$, Mean $( \pm$ SEM) percentage of freezing collapsed across the 8 min tone for the data and groups described in $A$ (NO PRE, striped bar; PRE-DIFF, black bar; PRE-SAME, white bar).

inhibition of conditional freezing is caused, in part, by the retrieval of conflicting cue memories (the CS-US memory acquired during training and the CS-no event memory acquired during preexposure) in the PRE-SAME group. Moreover, these results suggest that rats preexposed to the tone do in fact acquire the CS-US association to the extent that rats in the PRE-DIFF group express high levels of freezing to the tone when tested outside of the preexposure context. Therefore, latent inhibition arises because memories acquired during CS preexposure interfere with memories acquired during fear conditioning (Bouton, 1993), and as Experiment 1 demonstrates, context appears to mediate this interference.

\section{EXPERIMENT 2: DOES THE HIPPOCAMPUS MEDIATE CONTEXTUAL RETRIEVAL OF LATENT INHIBITION?}

Because of the role of the hippocampus in contextual learning, it is of considerable interest to determine whether it plays a role in contextual memory retrieval. In this regard, Honey and Good (1993) investigated the contextual specificity of latent inhibition using an appetitive conditioning procedure. They found that pretraining hippocampal lesions eliminated within-subject contextual specificity of latent inhibition, suggesting a role of the hippocampus in contextual memory retrieval. However, Honey and Good (1993) did not isolate retrieval processes per se, because they made permanent hippocampal lesions before any behavioral training. In the present experiment, we have overcome this problem by using reversible inactivation of the $\mathrm{DH}$ at the time of the retrieval test. Muscimol, a $\mathrm{GABA}_{\mathrm{A}}$ receptor agonist, was used to inactivate the DH temporarily (Bellgowan and Helmstetter, 1995; Mao and Robinson, 1998). Rats were treated as described in Experiment 1 except that they were implanted with bilateral guide cannulas above the $\mathrm{DH}$ before behavioral training and testing. Muscimol has proved to be a reliable agent for temporarily inactivating a number of brain structures including the amygdala (Helmstetter and Bellgowan, 1994; Muller et al., 1997) and hippocampus (Bellgowan and Helmstetter, 1995; Mao and Robinson, 1998). And, unlike permanent lesions, muscimol inactivation allowed us to target memory retrieval during an extinction test conducted $1 \mathrm{~d}$ after fear conditioning. [Note that one possible strategy for this experiment would have been to make permanent excitotoxic lesions in the $\mathrm{DH}$ immediately after fear conditioning. However, this would have forced us to interpose a 1-week delay (for surgical recovery) between fear conditioning and retrieval testing, and long retention intervals reduce latent inhibition (Bouton, 1993). The reversible lesion technique permitted us to assess latent inhibition within $24 \mathrm{hr}$ of fear conditioning.]

\section{MATERIALS AND METHODS}

Subjects. The subjects were 55 adult male Long-Evans rats (200-224 gm) obtained and housed as described in Experiment 1.

Surgery. One week before behavioral testing, the rats were implanted with stainless steel guide cannulas aimed at the DH. The rats were treated with atropine methyl nitrate $(0.4 \mathrm{mg} / \mathrm{kg}$, i.p.), anesthetized with sodium pentobarbital (Nembutal; $65 \mathrm{mg} / \mathrm{kg}$, i.p.), and mounted in a Kopf stereotaxic apparatus (Kopf Instruments, Tujunga, CA). The scalp was incised and retracted, and the head position was adjusted to place bregma and lambda in the same horizontal plane. Small burr holes $(1 \mathrm{~mm}$ in diameter) were drilled in the skull for bilateral placement of stainless steel guide cannulas (23 gauge; $12 \mathrm{~mm}$ in length; Small Parts, Miami Lakes, FL) into the $\mathrm{DH}(3.8 \mathrm{~mm}$ posterior to bregma, $2.5 \mathrm{~mm}$ lateral to bregma, and $1.8 \mathrm{~mm}$ ventral to dura) and three small jeweler's screws. Dental acrylic was used to affix the cannulas to the skull and to seal the scalp incision. After surgery, stainless steel obturators (30 gauge; $12 \mathrm{~mm}$ in length; Small Parts) were inserted into the guides. The obturators were removed and replaced every other day during the $7 \mathrm{~d}$ recovery period.

Apparatus and procedure. The behavioral apparatus was identical to that described in Experiment 1 . The rats were randomly assigned to a two $\times$ two (preexposure context $\times$ inf usion) design. For this design, rats underwent guide cannula implantation surgery $7 \mathrm{~d}$ after being housed in the vivarium. After surgery, the rats were allowed $7 \mathrm{~d}$ to recover before $\mathrm{CS}$ preexposure commenced. Rats were preexposed to the tone in either context $\mathrm{A}$ or $\mathrm{C}$, as described in Experiment 1 .

Twenty-four hours after the final day of CS preexposure, the rats were transported to the room where they would later be infused to familiarize them with both the environment and the infusion procedure. The goal was to obviate any potential effect that being placed in a new context for drug infusion might have on test-day performance. On arrival in the infusion room, the obturators were removed from the rats and replaced by 30 gauge injection cannulas (Small Parts), which extended into the guide cannulas but were short of the guides $12 \mathrm{~mm}$ length. The rats were 


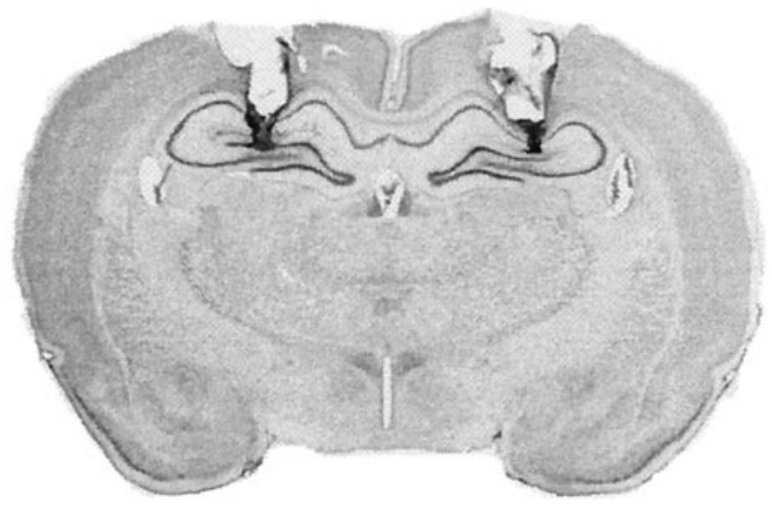

Figure 2. Cannula placement in the dorsal hippocampus. Photomicrograph showing a thionin-stained coronal section from the brain of a rat with representative cannula placements in the dorsal hippocampus. The darkly stained regions within the hippocampus indicate the injector track.

placed into brown plastic waste buckets whose bottoms were lined with corncob bedding. The injection cannulas were attached to polyethylene tubing (PE-10), which was in turn connected to $10 \mu$ l Hamilton syringes mounted in an infusion pump (Harvard Apparatus, South Natick, MA). The pump did not engage the syringe plungers during this mock infusion procedure but was allowed to run for $94 \mathrm{sec}$ to habituate the rats to the sound of the pump. While the rats were in the buckets, the experimenter prevented each rat from becoming entangled in the polyethylene tubing. The experimenter also distracted the rats with finger pokes to discourage grooming during the infusion, because grooming often dislodged the injection cannulas. Rats remained in the waste buckets for 1 min after the pump was shut off, after which they were removed. After removal from the buckets, the obturators were replaced, and rats were transported back to their home cages.

On the following day the rats underwent fear conditioning as described in Experiment 1. Twenty-four hours after fear conditioning, the rats were transported to the infusion room before retrieval testing. The procedure was similar to that of the sham infusion described above but differed in several key aspects. Injection cannulas, which extended $1 \mathrm{~mm}$ beyond the guide cannulas, were inserted into the $\mathrm{DH}$. Rats were administered an infusion of either physiological saline $(0.9 \%)$ or muscimol $(1 \mu \mathrm{g} / \mu \mathrm{l}$ dissolved in $0.9 \%$ saline; Sigma, St. Louis, MO). A total volume of $0.5 \mu \mathrm{l}$ was infused into each DH over a $94 \mathrm{sec}$ period; this yielded an inf usion of $0.5 \mu \mathrm{g}$ of muscimol into each $\mathrm{DH}$. This infusion protocol was anticipated to inactivate a $1 \mathrm{~mm}$ radius of the $\mathrm{DH}$, based on measurements of muscimol diffusion and 2-deoxyglucose activity (Martin, 1991). One minute was allowed after infusion for diffusion of the drug, after which the obturators were replaced and the rats were returned to their home cages. Forty-five to ninety minutes after the inf usion, the rats were transported to context A where they underwent a tone extinction test as described in Experiment 1. Freezing behavior was quantified as described in Experiment 1.

Histology. Histological verification of cannula location was performed after behavioral testing. Rats were perfused across the heart with $0.9 \%$ saline followed by $10 \%$ formalin solution. After extraction from the skull, the brains were post-fixed in $10 \%$ formalin solution for $2 \mathrm{~d}$ and in $10 \%$ formalin and $30 \%$ sucrose solution until sectioning. Coronal sections $(50$ $\mu \mathrm{m}$ thick; every section taken proximal to cannula tracts) were cut on a cryostat $\left(-19^{\circ} \mathrm{C}\right)$ and wet-mounted on glass microscope slides with $70 \%$ ethanol. After drying, the sections were stained with $0.25 \%$ thionin to visualize neuronal cell bodies.

Data analysis. Freezing behavior data were subjected to the same types of analyses described in Experiment 1.

\section{RESULTS}

\section{Histology}

A photomicrograph in Figure 2 of a thionin-stained coronal section illustrates a representative cannula placement in the DH. The injection cannula tip placements of all the animals included in the analysis are summarized in Figure 3 (one rat was excluded

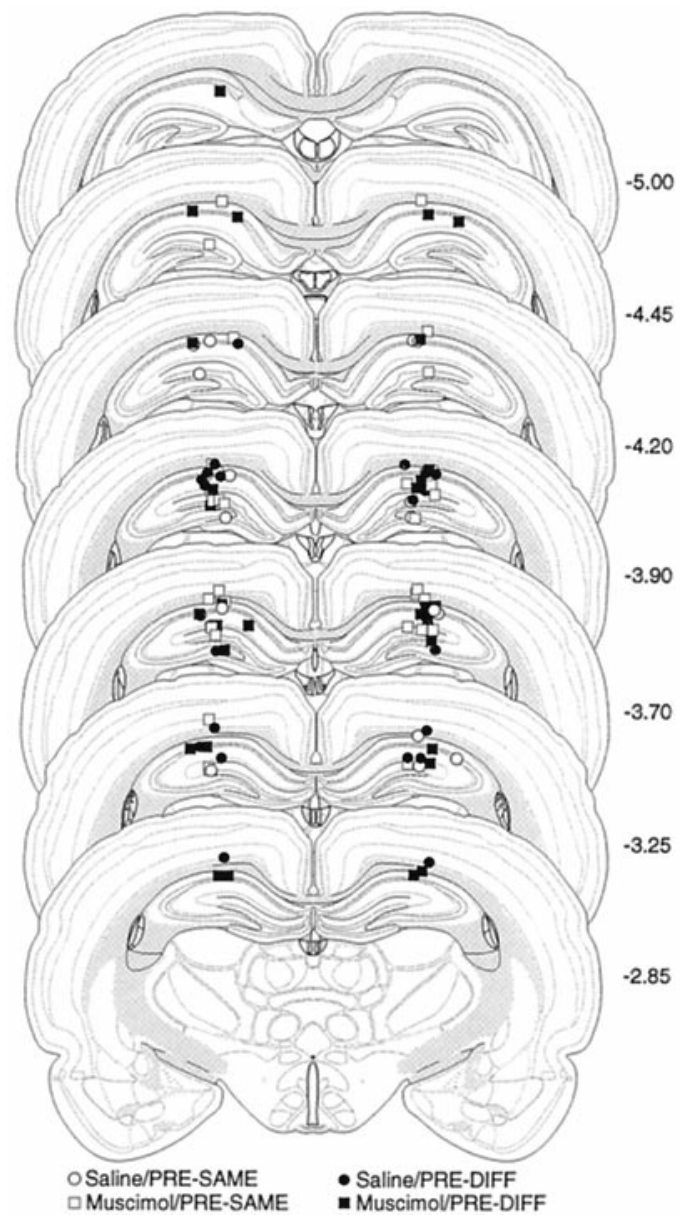

Figure 3. Schematic illustration of cannula placements in the dorsal hippocampus (Experiment 2). Schematic representation of injection cannula tip placements in the dorsal hippocampus for all rats included in the Experiment 2 analysis. The values to the right of each coronal section indicate the position of each section relative to bregma (millimeters caudal to bregma). Coronal brain section images are adapted from Swanson (1992).

from the analysis because of misplaced cannulas). This yielded the following groups: same-saline (SAME-SAL; $n=12$ ), samemuscimol (SAME-MUS; $n=16)$, DIFF-SAL $(n=11)$, and DIFF-MUS $(n=15)$. In all cases, the cannula placements were symmetrical and were localized throughout the rostrocaudal extent of the dorsal hippocampus. The placements did not consistently differ among groups. As stated above, we anticipated our inf usion protocol to inactivate no more than a $1 \mathrm{~mm}$ radius of the $\mathrm{DH}$, based on measurements of muscimol diffusion and 2-deoxyglucose activity (Martin, 1991). It is conceivable that cortical or thalamic tissue near the cannula tips was also inactivated, although previous estimates of muscimol spread would center the major area of inactivation within the $\mathrm{DH}$.

As shown in Figure 2, surgical implantation of the guide cannulas resulted in some damage to the cortex overlying the hippocampus, as well as compression of hippocampal tissue directly below the guide cannulas. This damage was restricted to the immediate region surrounding the guide cannulas and did not extend into the remainder of the DH. However, the pattern of behavior in saline controls (described below) was not different from that of unoperated rats in Experiment 1. This suggests that 
Figure 4. Dorsal hippocampal inactivation and the context-specific expression of latent inhibition (Experiment 2). $A$, Mean ( \pm SEM) percentage of freezing during the tone extinction test in rats preexposed in a context either the same $(S A M E)$ or different $(D I F F)$ from the test context (context A). Dorsal hippocampal infusions of either muscimol $(M U S)$ or saline $(S A L)$ were made $\sim 1$ $\mathrm{hr}$ before extinction testing. $B$, Mean ( \pm SEM) percentage of freezing collapsed across the $8 \mathrm{~min}$ tone for the data and groups described in $A$.

\section{(A) Tone extinction test}

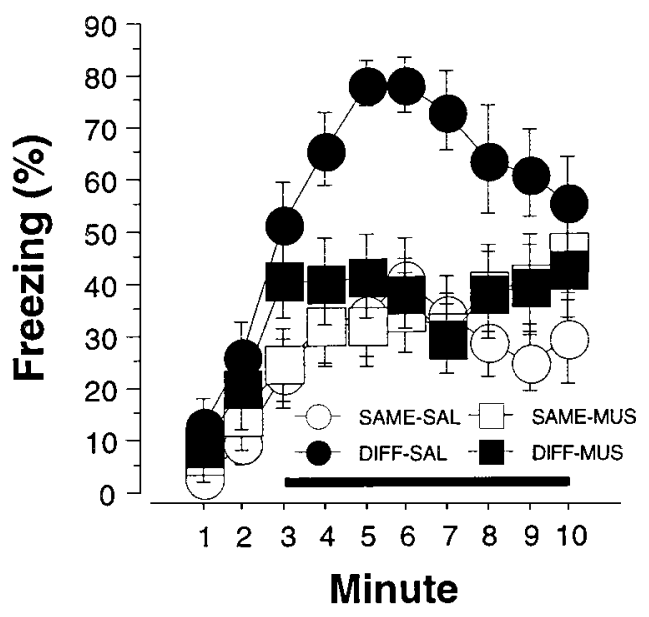

(B) Freezing during tone

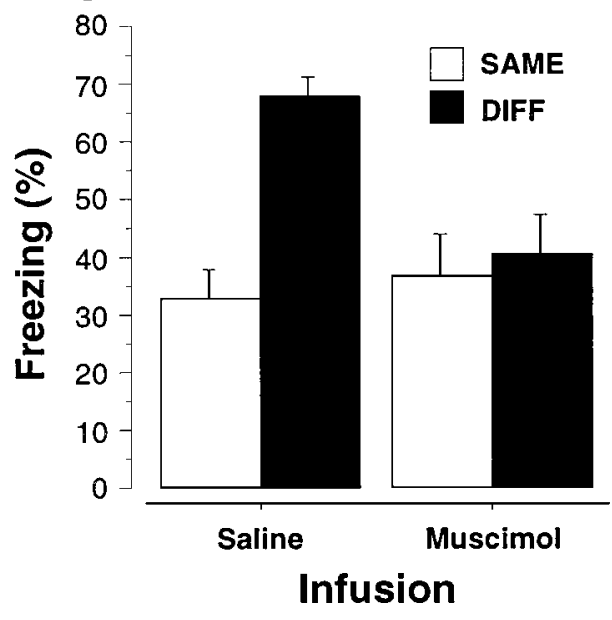

the cortical and hippocampal damage caused by guide cannula implantation was not sufficient to disrupt either fear conditioning or the context-specific expression of latent inhibition.

\section{Behavior}

As shown in Figure 4, $A$ and $B$, rats inf used with saline exhibited the same pattern of context-specific latent inhibition as did rats in Experiment 1. That is, saline rats preexposed and tested to the tone in the same context (SAME-SAL) displayed latent inhibition; they exhibited less freezing than did saline rats preexposed in a context that was different from that during testing (DIFFSAL). Most importantly, the contextual specificity of latent inhibition was not apparent in rats infused with muscimol in the $\mathrm{DH}$; these rats (SAME-MUS and DIFF-MUS) exhibited low levels of conditional freezing (i.e., latent inhibition) independent of whether the test context was the same as or different from that of preexposure. These impressions were confirmed by a significant main effect of preexposure context $\left[F_{(1,50)}=9.6 ; p<0.01\right]$ and a significant interaction of preexposure context and inf usion type $\left[F_{(1,50)}=6.0 ; p<0.02\right]$ in the ANOVA performed on the mean tone freezing data displayed in Figure $4 B$. Post hoc comparisons $(p<0.05)$ indicated that SAME-SAL rats froze significantly less than did SAME-DIFF rats, whereas rats infused with muscimol exhibited comparable and low levels of freezing independent of where they received tone preexposure. Thus, rats infused with muscimol exhibited latent inhibition regardless of the testing context. One inference of this result is that $\mathrm{DH}$ inactivation prevents the use of context as a retrieval cue.

\section{EXPERIMENT 3: DOES DORSAL HIPPOCAMPAL INACTIVATION PREVENT CONTEXTUAL DISCRIMINATION?}

Although Experiment 2 is consistent with a role of the $\mathrm{DH}$ in contextual retrieval, other interpretations are possible. For example, muscimol inf usion into the DH may impair the performance of conditional freezing. Another possibility is that DH inactivation may eliminate sensory-perceptual processing of contextual information without affecting contextual memory retrieval per se. Experiment 3 addressed these issues using a contextual discrimination design. If muscimol inf usion into the DH impairs freezing behavior or disrupts context processing, then contextual discrimination should not be possible after DH inactivation. For this experiment, the rats were administered unsignaled footshocks in one of two distinct contexts in which they were placed (context A or context B). During testing, the rats were returned to each context, and freezing was measured after inf usion of either saline or muscimol into the $\mathrm{DH}$. This provided a within-subjects measure of contextual discrimination of conditional freezing in the shock and no-shock contexts.

\section{MATERIALS AND METHODS}

Subjects, surgery, and behavioral apparatus. The subjects were 32 adult male Long-Evans rats (200-224 gm) obtained and housed as described in Experiment 1. All surgical procedures were the same as those described in Experiment 2. The conditioning chambers described in Experiment 1 comprised the behavioral apparatus.

Procedure. To examine the influence of hippocampal inactivation on contextual discrimination, a within-subjects design was used with a between-subjects factor of infusion (saline or muscimol) and a withinsubjects factor of context (shock or no shock) $(n=16$ per group). After a $7 \mathrm{~d}$ recovery from surgery, the rats were transported to the laboratory and familiarized with the infusion room as described in Experiment 2. One day later, the $2 \mathrm{~d}$ conditioning phase of Experiment 3 commenced. On the first day of training, half of the rats were placed in context A (shock context) and were presented with five unsignaled footshocks (2 sec; $1.0 \mathrm{~mA} ; 3$ min preshock period; 1 min intershock interval). The other half of the rats were placed into context B (no-shock context) and remained there for $8 \mathrm{~min}$ before being returned to their home cage. On the second day of training, the rats received training in the context that they did not experience on the first day. Thus, at the end of the 2 training days, each rat had been shocked in one context and merely exposed to another.

The $2 \mathrm{~d}$ testing phase began the day after training. Before extinction testing, the rats were infused with either muscimol or saline solution as described in Experiment 2. Forty-five to ninety minutes after the infusion, half of the rats were placed into context $\mathrm{A}$, and freezing was recorded for $8 \mathrm{~min}$ as described in Experiment 1. The other half of the rats were placed in context $\mathrm{B}$ for $8 \mathrm{~min}$, and freezing behavior was recorded. Forty-eight hours later, the rats were returned to the laboratory, infused with either muscimol or saline (each rat received the same type of infusion that was received before the first extinction test), and placed in the context that was different from the one they had been in $2 \mathrm{~d}$ earlier; freezing was recorded as described above. Thus, for both training and testing, exposure to the experimental contexts was counterbalanced such that a given rat would experience the contexts in one of the following orders: [(training contexts-testing contexts) $\mathrm{AB}-\mathrm{AB}, \mathrm{AB}-\mathrm{BA}$, $\mathrm{BA}-\mathrm{AB}$, and $\mathrm{BA}-\mathrm{BA}]$. This allowed us to establish a within-subjects measure of contextual discrimination.

Histology and data analysis. Histological verification of cannula placement and data analysis were performed as described in Experiment 2. 


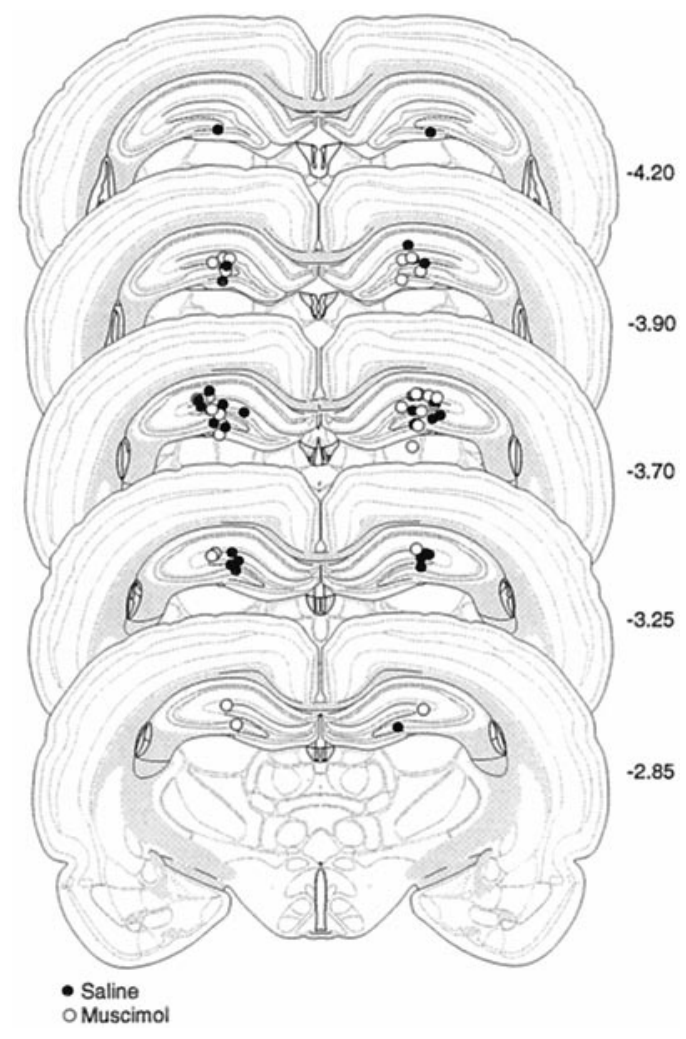

Figure 5. Schematic illustration of cannula placements in the dorsal hippocampus (Experiment 3). Schematic representation of injecting cannula tip placements in the dorsal hippocampus for all rats included in the Experiment 3 analysis. Values to the right of the coronal sections indicate the position of each section relative to bregma (millimeters caudal to bregma). Coronal brain section images are adapted from Swanson (1992).

\section{RESULTS}

\section{Histology}

Injection cannula tip placements of all the animals included in the analysis are summarized in Figure 5. As in Experiment 2, the placements were symmetrical and did not differ consistently between groups.

\section{Behavior}

Freezing behavior was not influenced by the order in which rats were trained or tested in contexts $\mathrm{A}$ and $\mathrm{B}$, so this variable was eliminated from the analyses described below. Inspection of Figure 6 shows that rats infused with either saline or muscimol froze significantly more when tested in the shock context than when they were tested in the no-shock context. This indicates that the rats learned to discriminate the contexts reliably. This observation was confirmed by a significant main effect of test context $\left[F_{(1,30)}=103.0 ; p<0.0001\right]$ in the ANOVA. It is also apparent that rats infused with muscimol exhibit more freezing than do rats infused with saline in either testing context. This observation was confirmed by a significant main effect of infusion type $\left[F_{(1,30)}\right.$ $=6.4 ; p<0.02]$ in the ANOVA. Importantly, however, muscimol infusion into the DH did not effect contextual discrimination. That is, muscimol rats exhibited significantly greater levels of freezing in the shock context compared with the no-shock context.

These results suggest that the low levels of conditional responding in the muscimol-infused rats in Experiment 2 were not caused by performance deficits or a general impairment in contextual

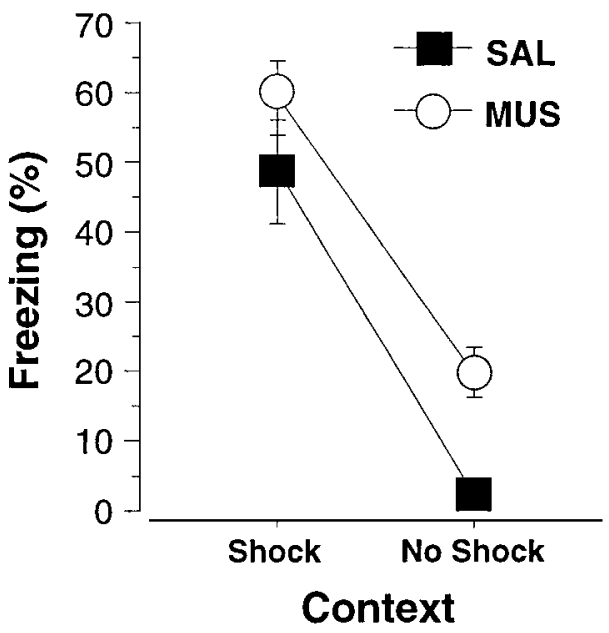

Figure 6. Dorsal hippocampal inactivation and expression of a contextual discrimination (Experiment 3). Mean ( \pm SEM) percentage of freezing in a context paired with footshock (Shock) and a context not paired with footshock (No Shock) in rats that received dorsal hippocampal inf usions of either muscimol (MUS) or saline $(S A L) \sim 1 \mathrm{hr}$ before extinction testing. The test order for each context was counterbalanced, and the tests were conducted 1 and $2 \mathrm{~d}$ after discrimination training. This yielded a within-subjects measure of contextual discrimination.

processing. Thus, the ability of the muscimol rats in Experiment 3 to perform the contextual discrimination suggests that muscimol only disrupts contextual retrieval in situations in which the meaning of a cue or context is ambiguous (as in Experiment 2). Collectively, these data bolster emerging evidence that the $\mathrm{DH}$ may not be essential for first-order contextual learning (e.g., Maren and Fanselow, 1997; Maren et al., 1997) but may be involved in higher-order contextual processes, including encoding stimulus conjunctions (e.g., Rudy and Sutherland, 1995), processing incidental contextual information (e.g., Good et al., 1998), and contextual memory retrieval (e.g., Honey and Good, 1993).

\section{DISCUSSION}

In the present experiments, we isolated a retrieval process in latent inhibition (Experiment 1) and examined the effects of $\mathrm{DH}$ inactivation during that process (Experiment 2). We found that the expression of latent inhibition was context specific and that muscimol inactivation of the DH eliminated this contextual specificity. We demonstrated that our method of hippocampal inactivation did not intrude on the rat's ability to make a simple contextual discrimination nor did it attenuate freezing behavior in general (Experiment 3). Together these experiments indicate that a functional hippocampus is required for contextual memory retrieval in a latent inhibition paradigm. These results offer insight into both the nature and causes of latent inhibition and the role of the hippocampus in memory processes.

With regard to the nature of latent inhibition, our experiments sustain the claim of Bouton (1993) that an important component of latent inhibition involves retrieval, as compared with encoding processes. That is, in agreement with Bouton and Swartzentruber (1989), we have shown that despite comparable CS preexposure, latent inhibition was only expressed by rats that were tested in the preexposure context; rats that received CS preexposure in a different context did not exhibit latent inhibition. Dexter and Merrill (1969) and Wright et al. (1986) have reported similar results. These data are not consistent with models of latent inhibition that posit a reduction in CS associability because of 
context CS association (Wagner, 1978, 1981) or decrements in attention acquired during the preexposure phase (Mackintosh, 1975), because neither of these models can account for the context-specific expression of latent inhibition.

It is well established that the hippocampus is involved in mediating latent inhibition in several paradigms (Ackil et al., 1969; Solomon and Moore, 1975; McFarland et al., 1978; Kaye and Pearce, 1987; Han et al., 1995). In these paradigms, DH lesions attenuate or eliminate latent inhibition when preexposure, training, and testing are conducted in the same context. These data are typically interpreted in terms of a failure to decrease CS associability during the CS preexposure phase in rats with $\mathrm{DH}$ lesions. However, permanent DH lesions might also exert their effect by disrupting contextual retrieval during testing. In agreement with this view, we have demonstrated in Experiment 2 that reversible inactivation of the $\mathrm{DH}$ during testing eliminates the context specificity of latent inhibition. Although our data do not rule out a role of the hippocampus in decremental associative processes (e.g., Han et al., 1995), they do suggest that the effects of DH lesions on latent inhibition may be the result of a disruption in retrieval processes.

The important role of retrieval processes in latent inhibition and the involvement of the hippocampus in these processes force a reconceptualization of the role of the hippocampus in this form of learning. An important issue raised by the present study concerns the role of the hippocampus in the acquisition versus the expression of latent inhibition. Because most studies have used permanent pretraining lesions of the hippocampus, there is a great deal of data indicating that hippocampal lesions impair the acquisition of latent inhibition (Ackil et al., 1969; Solomon and Moore, 1975; McFarland et al., 1978; Kaye and Pearce, 1987; Han et al., 1995). That is, rats with hippocampal lesions tend not to exhibit attenuate conditional responding after preexposure to the to-be-CS. However, as we have shown presently, hippocampal inactivation during retrieval testing does not impair the expression of latent inhibition per se; it merely eliminates the contextspecific expression of latent inhibition. In our experiments, rats without a functional hippocampus are in fact more likely to exhibit reduced conditional responding than are intact rats, at least in contexts that are different from those of preexposure.

We suggest that this pattern of results indicates that there are at least two distinct learning processes in the latent inhibition paradigm that require the hippocampus. The associative processes we presume to be operating during latent inhibition are based on a model of extinction described by Bouton (1994) and are illustrated in Figure 7. We posit that the first process, which has been targeted by most permanent lesion studies, consists of the acquisition of a CS-no event memory during the CS preexposure phase of latent inhibition training. We suggest that rats with hippocampal lesions do not encode a CS-no event representation during the CS preexposure phase of training. Hence, they do not learn that the CS has no consequence or that the CS is "insignificant." The failure to form such a representation might be indexed by the failure of rats with hippocampal lesions to decrement their orienting response to the CS during preexposure (Kaye and Pearce, 1987). Although we believe that the hippocampus is required to encode CS-no event associations, we propose that this representation is not stored in the hippocampus.

The second hippocampus-dependent process we posit for latent inhibition is contextual memory retrieval (e.g., Hirsh, 1974), which functions to retrieve the CS-no event association (presumed to reside outside of the hippocampus) in the context of

\section{Intact}

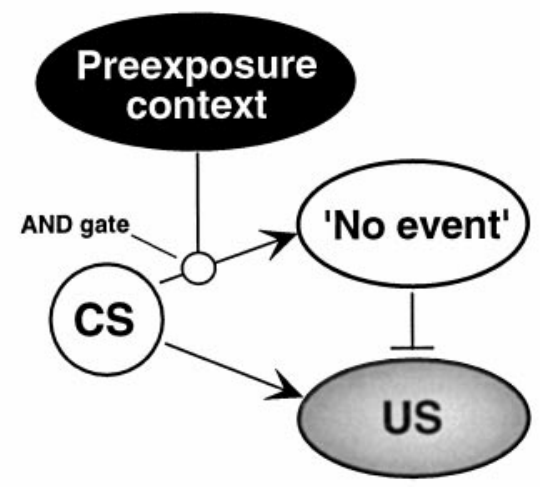

DH Inactivation

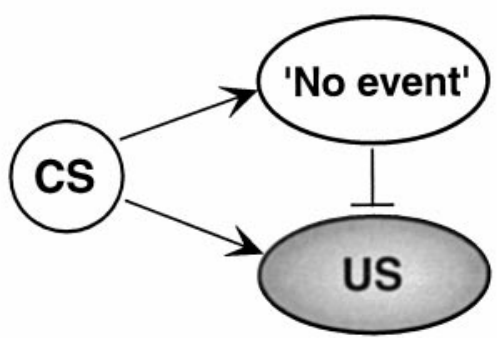

Figure 7. Model of latent inhibition. We have elaborated Bouton's extinction model (Bouton, 1994) to conceptualize contextual retrieval in latent inhibition. We propose that a CS-no event association is acquired during CS preexposure and that this association is only retrieved in the presence of the contextual stimuli that were associated with preexposure. In other words, an "AND gate" is interposed between the CS and no-event representations, and this gate allows contextual retrieval cues to regulate the expression of the $\mathrm{CS}-$ no event association. The expression of the excitatory CS-US association, which is acquired during conditioning, is context independent. During extinction testing in the preexposure context, competition between the active CS-no event association and the CS-US association results in a suppression of the CS-US memory, favoring performance of the CS-no event memory. In this way, latent inhibition is only expressed in the context of preexposure. This allows for the expression of latent inhibition to be context specific. Our inactivation data suggest that the hippocampus allows context to regulate the expression of the CS-no event memory via the AND gate. In the absence of a functional hippocampus, contextual retrieval cues cannot regulate expression of the CS-no event memory. As a result, expression of the CS-no US memory becomes context independent, and conditional responding to the CS is limited in all test contexts.

preexposure. As illustrated in Figure 7, hippocampal inactivation at the time of contextual retrieval removes contextual gating of the CS-no event memory and allows this memory to be expressed in a context-independent manner (diminished conditional responding is observed in all test contexts). In this way, context may be serving as an occasion setter in a manner that is analogous to how a discrete feature-positive stimulus facilitates or retrieves its response in a typical occasion-setting experiment (e.g., Holland, 1983). In fact hippocampal lesions have been reported to disrupt acquisition and performance of this type of learning (e.g., Ross et al., 1984).

This two-process model of latent inhibition accounts for impairments in the acquisition of latent inhibition after pretraining hippocampal lesions and the preserved, yet context-independent, latent inhibition after hippocampal inactivation during retrieval testing. This model predicts that permanent hippocampal lesions 
made after $\mathrm{CS}$ preexposure, but before conditioning or retrieval testing, should eliminate the context specificity of latent inhibition without affecting the magnitude of latent inhibition per se. It also predicts that reversible inactivation of the hippocampus during CS preexposure should eliminate the acquisition of latent inhibition. Such experiments have yet to be performed.

The selective involvement of the $\mathrm{DH}$ in contextual retrieval, as opposed to either contextual processing or memory retrieval per se, is demonstrated by our third experiment. In this experiment, reversible inactivation of the $\mathrm{DH}$ did not prevent retrieval of a context-US association, nor did it affect the expression of a contextual discrimination. These results were somewhat surprising because others have shown previously that $\mathrm{DH}$ lesions made shortly after training attenuate contextual freezing (Kim and Fanselow, 1992; Maren et al., 1997; Anagnostaras et al., 1999). Moreover, Bellgowan and Helmstetter (1995) have reported that reversible inactivation of the $\mathrm{DH}$ eliminates the expression of contextual freezing. The reason for this discrepancy is not clear, but the extent of $\mathrm{DH}$ inactivation in our experiments may have been insufficient to yield deficits in contextual discrimination, despite the robust impairment it caused in the context specificity of latent inhibition. It is also possible that muscimol inactivation, although probably resulting in a smaller tissue disruption than hippocampal lesions, may have been less specific for the hippocampus. That is, cortical or thalamic tissue in the vicinity of the guide cannulas may have been affected by the infusion. This ancillary inactivation may have offset the influence of $\mathrm{DH}$ inactivation.

Our interpretations of the role of the $\mathrm{DH}$ in contextual retrieval join those from disparate sources that implicate hippocampal function in the retrieval process. For instance, recent human neuroimaging studies reveal hippocampal formation activation during performance of tasks specialized to examine retrieval (Squire et al., 1992; Gabrieli et al., 1997; Maguire et al., 1997). Furthermore, post-training lesions or muscimol inactivation of the DH disrupts retrieval of spatial memory in a Morris water maze task in rats (Moser and Moser, 1998). In addition, Freeman et al. (1997) have found that lesions of the entorhinal cortex, a major hippocampal afferent, disrupt the ability of novel contexts to modulate extinction performance in rabbits. Moreover, CSevoked multiunit activity in the hippocampal area CA1 during concurrently trained approach and avoidance tasks is specific to the contingency predicted by task context (Freeman et al., 1996). Together, these data are consistent with the role of the hippocampus in contextual memory retrieval.

In conclusion, context has been demonstrated to be a strong modulator of performance of learned behavior in both animals (Spear, 1973) and humans (Tulving and Thomson, 1973). Thus, understanding the substrates underlying contextual retrieval processes may elucidate the mechanisms governing much of behavior. Several attempts have been made to examine the relationships among context, retrieval, and the hippocampus using associativelearning models (e.g., Good and Honey, 1991; Penick and Solomon, 1991; Honey and Good, 1993). And although in-roads have been made, permanent hippocampal lesions before training confound the effects of the lesions on encoding with their effects on retrieval processes. Use of reversible lesions, like those induced by muscimol microinf usion, together with behavioral procedures that specifically target various stages of memory (i.e., encoding, consolidation, and retrieval) may yield a sharper picture of the functional architecture of memory systems.

\section{REFERENCES}

Ackil JE, Mellgren RL, Halgren C, Frommer GP (1969) Effects of CS preexposures on avoidance learning in rats with hippocampal lesions. J Comp Physiol Psychol 69:739-747.

Anagnostaras S, Maren S, Fanselow M (1999) Temporally graded retrograde amnesia of contextual fear after hippocampal damage in rats: within-subjects examination. J Neurosci 19:1106-1114.

Bellgowan PSF, Helmstetter FJ (1995) Effects of muscimol applied to the dorsal hippocampus on the acquisition and expression of cued versus contextual fear conditioning. Soc Neurosci Abstr 21:1213.

Bouton ME (1993) Context, time, and memory retrieval in the interference paradigms of Pavlovian learning. Psychol Bull 114:80-99.

Bouton ME (1994) Context, ambiguity, and classical conditioning. Curr Dir Psychol Sci 3:49-53.

Bouton ME, Swartzentruber D (1989) Slow reacquisition following extinction: context, encoding, and retrieval mechanisms. J Exp Psychol Anim Behav Process 15:43-53.

Dexter WR, Merrill HK (1969) Role of contextual discrimination in fear conditioning. J Comp Physiol Psychol 69:677-681.

Fanselow MS (1980) Conditional and unconditional components of postshock freezing. Pavlov J Biol Sci 15:177-182.

Freeman Jr JH, Cuppernell C, Flannery K, Gabriel M (1996) Contextspecific multisite cingulate cortical, limbic thalamic, and hippocampal neuronal activity during concurrent discriminative approach and avoidance training in rabbits. J Neurosci 16:1538-1549.

Freeman Jr JH, Weible A, Rossi J, Gabriel M (1997) Lesions of the entorhinal cortex disrupt behavioral and neuronal responses to context change during extinction of discriminative avoidance behavior. Exp Brain Res 115:445-457.

Gabrieli JDE, Brewer JB, Desmond JE, Glover GH (1997) Separate neural bases of two fundamental memory processes in the human medial temporal lobe. Science 276:264-266.

Good M, Honey RC (1991) Conditioning and contextual retrieval in hippocampal rats. Behav Neurosci 105:499-509.

Good M, de Hoz L, Morris RGM (1998) Contingent versus incidental context processing during conditioning: dissociation after excitotoxic hippocampal plus dentate gyrus lesions. Hippocampus 8:147-159.

Hall G, Minor H (1984) A search for context-stimulus associations in latent inhibition. Q J Exp Psychol B 36:145-169.

Han J-S, Gallagher M, Holland P (1995) Hippocampal lesions disrupt decrements but not increments in conditioned stimulus processing. J Neurosci 15:7323-7329.

Helmstetter FJ, Bellgowan PS (1994) Effects of muscimol applied to the basolateral amygdala on acquisition and expression of contextual fear conditioning in rats. Behav Neurosci 108:1005-1009.

Hirsh R (1974) The hippocampus and contextual retrieval of information from memory: a theory. Behav Biol 12:421-444.

Hirsh R (1980) The hippocampus, conditional operations, and cognition. Physiol Psychol 8:175-182.

Holland PC (1983) Occasion setting in Pavlovian feature positive discriminations. In: Quantitative analyses of behavior: discrimination processes (Commons ML, Herrnstein RJ, Wagner AR, eds), pp 183-206. Cambridge, MA: Ballinger.

Holland PC, Bouton ME (1999) Hippocampus and context in classical conditioning. Curr Opin Neurobiol 9:195-202.

Honey RC, Good M (1993) Selective hippocampal lesions abolish the contextual specificity of latent inhibition and conditioning. Behav Neurosci 107:23-33.

Kaye H, Pearce JM (1987) Hippocampal lesions attenuate latent inhibition and the decline of the orienting response in rats. Q J Exp Psychol B 39:107-125.

Kim JJ, Fanselow MS (1992) Modality-specific retrograde amnesia of fear. Science 256:675-677.

Lubow RE (1973) Latent inhibition. Psychol Bull 79:398-407.

Mackintosh NJ (1975) A theory of attention: variations in the associability of stimuli with reinforcement. Psychol Rev 4:276-298.

Maguire EA, Frackowiak RSJ, Frith CD (1997) Recalling routes around London: activation of the right hippocampus in taxi drivers. J Neurosci 17:7103-7110.

Mao JB, Robinson JK (1998) Microinjection of GABA-A agonist muscimol into the dorsal but not the ventral hippocampus impairs nonmnemonic measures of delayed non-matching-to-position performance in rats. Brain Res 784:139-147.

Maren S (1998) Overtraining does not mitigate contextual fear condi- 
tioning deficits produced by neurotoxic lesions of the basolateral amygdala. J Neurosci 18:3088-3097.

Maren S, Fanselow MS (1997) Electrolytic lesions of the fimbria/fornix, dorsal hippocampus, or entorhinal cortex produce anterograde deficits in contextual fear conditioning in rats. Neurobiol Learn Mem 67:142-149.

Maren S, Aharonov G, Fanselow MS (1997) Neurotoxic lesions of the dorsal hippocampus and Pavlovian fear conditioning in rats. Behav Brain Res 88:261-274.

Maren S, Anagnostaras SG, Fanselow MS (1998) The startled seahorse: is the hippocampus necessary for contextual fear conditioning? Trends Cog Sci 2:39-42.

Martin JH (1991) Autoradiographic estimation of the extent of reversible inactivation produced by microinjection of lidocaine and muscimol in the rat. Neurosci Lett 127:160-164.

McFarland DJ, Kostas J, Drew WG (1978) Dorsal hippocampal lesions: effects of preconditioning CS exposure on flavor aversion. Behav Biol 22:398-404.

Moser MB, Moser EI (1998) Distributed encoding and retrieval of spatial memory in the hippocampus. J Neurosci 18:7535-7542.

Muller J, Corodimas KP, Fridel Z, LeDoux JE (1997) Functional inactivation of the lateral and basal nuclei of the amygdala by muscimol infusion prevents fear conditioning to an explicit conditioned stimulus and to contextual stimuli. Behav Neurosci 111:683-691.

Penick S, Solomon PR (1991) Hippocampus, context and conditioning. Behav Neurosci 105:611-617.

Phillips RG, LeDoux JE (1992) Differential contribution of amygdala and hippocampus to cued and contextual fear conditioning. Behav Neurosci 106:274-285.
Ross RT, Orr WB, Holland PC, Berger TW (1984) Hippocampectomy disrupts acquisition and retention of learned conditional responding. Behav Neurosci 98:211-225.

Rudy JW, Sutherland RJ (1995) Configural association theory and the hippocampal formation: an appraisal and reconfiguration. Hippocampus 5:375-389.

Solomon PR, Moore JW (1975) Latent inhibition and stimulus generalization of the classically conditioned nictitating membrane response in rabbits (Oryctolagus cunniculus) following dorsal hippocampal ablation. J Comp Physiol Psychol 89:1192-1203.

Spear NE (1973) Retrieval of memory in animals. Psychol Rev 80:163-194.

Squire LR, Ojemann JG, Miezin FM, Petersen SE, Videen TO, Raichle ME (1992) Activation of the hippocampus in normal humans: a functional anatomical study of memory. Proc Natl Acad Sci USA 89:1837-1841.

Swanson LW (1992) Brain maps: structure of the rat brain. New York: Elsevier.

Tulving E, Thomson DM (1973) Encoding specificity and retrieval processes in episodic memory. Psychol Rev 80:352-373.

Wagner AR (1978) Expectancies and the priming of STM. In: Cognitive processes in animal behavior (Hulse SH, Fowler H, Honig WK, eds), pp 177-209. Hillsdale, NJ: Erlbaum.

Wagner AR (1981) SOP: a model of automatic memory processing in animal behavior. In: Information processing in animals: memory mechanisms (Spear NE, Miller RR, eds), pp 5-47. Hillsdale, NJ: Erlbaum.

Wright DC, Skala KD, Peuser KA (1986) Latent inhibition from context dependent retrieval of conflicting information. Bull Psychon Soc 24: $152-154$. 